\title{
Editorial
}

\section{Las nodrizas y su importancia en los cuidados}

\author{
Wet nurses and their importance in care
}

\section{As amas de leite e sua importância no cuidado}

\author{
Antonio Martínez Sabater \\ Doctor en Enfermería. Profesor Colaborador. Departament d'Infermeria i Podologia de la Universitat de València. \\ Cómo citar esta editorial en edición digital: Martínez Sabater, A. (2014) Las nodrizas y su importancia en los cuidados. Cultura \\ de los Cuidados (Edición digital) 18, 40. Disponible en: http://dx.doi.org/10.7184/cuid.2014.40.01> \\ Correspondencia: Facultat d'Infermeria i Podologia. Universitat de València. C/Jaume Roig s/n. 46010. Valencia.
} Correo electrónico: Antonio.Martinez-Sabater@uv.es

Recibido: 1/07/2014; Aceptado: 15/09/2014

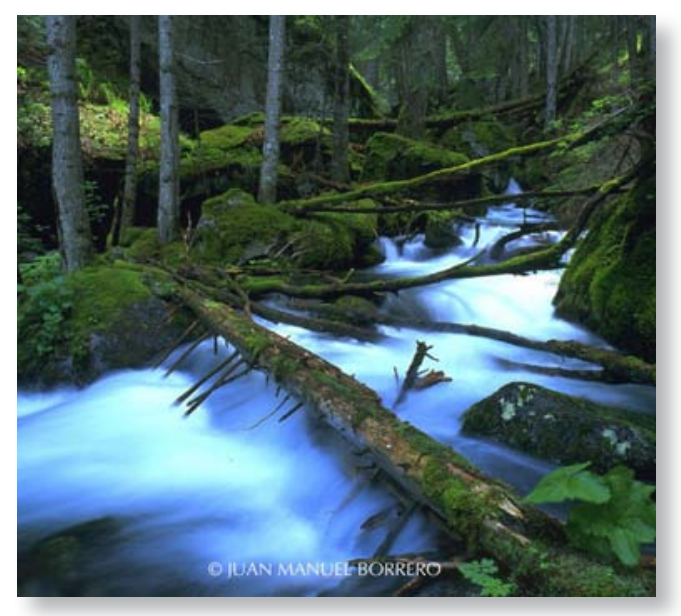

La enfermería como actividad del cuidado se encuentra ligada a la aparición de la humanidad, habiéndose desarrollado en paralelo a ella. Hasta llegar a la profesionalización existente en la actualidad se ha seguido un largo camino en el que se ha ido sucediendo diferentes figuras preprofesionales, competencias $\mathrm{y}$ funciones entre las que podemos encontrar las sanadoras, parteras y las nodrizas que con el devenir de los años han ido desapareciendo y transformándose. Como ya indicaba Virginia Henderson, a la enfermera se la ha llamado "la madre profesional", y, en efecto, como una madre que responde a las necesidades de su hijo, a veces debe realizar tareas que son ajenas a su profesión (Henderson, 1971), y podríamos incluso concretar que en cada momento histórico y situación concreta las actividades y funciones de la enfermera se han ido adaptando al momento social. Una de las figuras que tradicionalmente podremos relacionar con los cuidados en los diferentes momentos de nuestra profesión es la nodriza, que como indica Siles y col., etimológicamente proviene del término latino nutricia (que eran los pagos o salarios entregados a la nodriza por su ocupación), del que se desarrollarán posteriormente términos como nutrix, nutrire, nurse,... que constatan el carácter biológico de las actividades y la relación simbólica de los cuidados con las actividades nutríticas (Siles, y otros, 1998).

Los roles biológicos de la mujer que la hace encargada de las actividades de lactancia y crianza, y esta como elemento funcional dentro del marco del hogar provocará que se produzca una transformación de éstos en pseudooficios, oficios y profesiones (nodriza, criada, maestra, enfermera) y en actividades marginales remuneradas como la prostitución (Siles González, Pasado, presente y futuro de la enfermería en España: perspectiva histórica y epistemológi- 
ca, 1996). Concepto biologista establecido por una diferencia de género, fruto del proceso socializador que ha responsabilizado a la mujer de las funciones reproductivas y al hombre del trabajo productivo, que ha supuesto a lo largo de la historia una desigual posición de hombres y mujeres en la sociedad, minusvalorando el trabajo reproductivo respecto al trabajo productivo (Larrañaga, Arregui, \& Arpal, 2004). Así, la nodriza supuso la profesionalización del trabajo reproductivo, caracterizado por ser eminentemente femenino, no estar remunerado y permanecer invisible incluso a los ojos de las personas que lo llevan a cabo (Carrasquer, Toms, Tejero, \& Romero, 1998) y permitió la salida del hogar de la mujer que si bien no participaban en el proceso productivo industrial, aprovechaba un recurso natural como el de la leche con el fin de obtener un beneficio económico, permitiendo la función de la reproducción de la fuerza del trabajo (Fuentes Caballero, 1996). Al mismo tiempo, este rol laboral supuso un desarrollo económico y social del grupo familiar, propiciando emigraciones continuas de nodrizas a la ciudad, y cuya labor va a influir en el desarrollo económico de la zona de partida debido al importante flujo económico (Soler, 2010), pese a que debe tenerse en cuenta que al asimilarse a la esfera privada de producción y reproducción de las condiciones de vida, se producía una invisibilización de la mujer siendo únicamente valoradas por os productos finales (hijos criados, comida elaborada), y obviando todas las actividades, tanto dentro como fuera del hogar (cocineras, lavanderas, etc.) que permitían el desarrollo familiar (Herrera Feria, 2003). Así la lactancia mercenaria se convirtió en uno de los nichos de trabajo de estas mujeres en diferentes ámbitos: las nodrizas de las inclusas, las nodrizas que crían en sus propias casas y las mujeres que ejercen la lactancia asalariada en su propia casa (Soler, 2010), ocupando más de la cuarta parte de anuncios de ofertas de puestos de trabajo en los periódicos españoles del siglo XVIII (Paricio Talayero, 2004). En este proceso laboral se va a producir una evolución de la figura, si bien en algunos países se había iniciado como un servicio altruista hacia los padres que han tenido que emigrar, siendo la nodriza una mujer de la zona rural, pasando al siglo XIX a ser una figura más dentro del servicio doméstico que vive y realiza su función en el hogar familiar (Fildes, 1988) (Mander, 2003). Esta evolución va a ser similar en otras situaciones, no estando inicialmente la tarea de amamantar restringida a las profesionales, sino que se establecen redes de apoyo entre las mujeres del grupo, convirtiéndose la profesionalización de la crianza en uno de los medios que permitió a las mujeres pudientes liberarse del vínculo constante al cuidado doméstico que caracteriza al papel femenino en las sociedades patriarcales (Expósito González, Rubio Pilarte, \& Solórzano Sánchez, 2012).

En la historia de la humanidad, la leche materna ha sido el único alimento que el recién nacido ha podido tomar para sobrevivir, convirtiéndose la lactancia materna en una actividad cultural que afecta a los mujeres en función de las creencias, clase social y nivel educativo entre otros. (Hernández Gamboa, 2008) (Riverón Corteguera, 1995). Teniendo en cuenta la necesidad y la importancia de la lactancia materna, y la falta de un sustituto eficaz, y dada la elevada mortalidad del uso de alimentación artificial, el pecho de la madre, o de la nodriza en las situaciones en las que ésta era la opción, suponía la diferencia entre la vida y la muerte del recién nacido (Paricio Talayero, 2004). Así, podríamos definir a la nodriza como la mujer que amamanta a un 
lactante que no es su hijo, hoy en desuso en la mayor parte de Occidente, el empleo de amas de cría se remonta a la Prehistoria y fue común hasta el siglo XIX para alimentar a niños cuyas madres no podían o no deseaban hacerlo. Así, el motivo de esta elección podían ser razones físicas como la insuficiente producción de leche, existencia de partos múltiples, o sociales, al permitir disminuir el período entre embarazos o simplemente librarse de una tarea percibida como socialmente inadecuada para las clases superiores (Expósito González, Rubio Pilarte, \& Solórzano Sánchez, 2012). Como indica Fuentes Caballero, "estas mujeres no hicieron otra cosa que buscar la solución que más se ajustaba a sus necesidades o deseos" (Fuentes Caballero, 1996).

Las nodrizas han sido un referente en el desarrollo social a través de la historia, apareciendo disposiciones sobre ellas en los códigos babilónicos (Leyes de Esnunna y Código de Hammurabi) (Yalom, 1997), siendo imprescindibles en las culturas clásicas en la que la mayor parte de mujeres de la nobleza recurrían a ellas (Paricio Talayero, 2004) y apareciendo referencias a las nodrizas en la Biblia tanto en el ámbito público como privado (BenLun, 2006). Al mismo tiempo será un referente en las culturas islámicas (Ruf-Nabhan, 2000) (Koçtürk T. , 92) (Koçtürk T. , 2003) y orientales (Botton Beja, 2000) (Macfarlane, 2002). En Europa, la lactancia mercenaria será predominante en la edad media sobre todo en las clases elevadas (Paricio Talayero, 2004), (Fildes, 1988), desarrollándose en Francia en el siglo XVIII las primeras legislaciones de protección a las niños amamantados por nodrizas, trabajo que como indica Fildes recibirá una compensación mayor que otros trabajos femeninos (Paricio, 2004), (Fildes, 1988). La lactancia mercenaria se extenderá por Europa durante el
Renacimiento y por diferentes clases sociales, así si en el siglo XVI era una práctica exclusiva de la aristocracia, se extiende en el XVII a la burguesía y alcanza en el XVIII a las clases populares, suponiendo un desarrollo de la industria de las nodrizas que suponía en 1780 que de 21.000 niños nacidos en Paris, 1.801 son amamantados por sus madres, 19.000 por una nodriza en el domicilio familiar, nourrice sur lieu, o en la inclusa y 199 en casa de una nodriza, generalmente en el campo, y que había supuesto en 1769 la creación del Bureau des Nourrices, Oficina de Nodrizas, dependiente del gobierno, (Paricio, 2004).

Pese a que ya en algunos textos medievales ya aparecía la lactancia mercenaria como problema, será a partir de la publicación del "Emile" de Rousseau cuando se inicia un proceso de construcción social que enuncia un ideal femenino de dedicación al hogar y a la maternidad que repercutirá en los comportamientos y actitudes hacia la vida familiar (Fuentes Caballero, 1996) (Bolufer Peruga, 1992), propugnándose una "vuelta a la naturaleza" y una vida familiar, que se vehiculizará a través de la literatura médica, divulgativa y la prensa con el fin de cambiar los hábitos (Bolufer Peruga, 1992) en el que se incidirá en que la descarga de la lactancia en nodrizas contradice el carácter natural del instinto maternal y las obligaciones inherentes a la maternidad biológica que era una obligación considerada como sagrada, convirtiéndose la transgresión en la falta más grave que puede cometer una mujer (Fuentes Caballero, 1996). Este discurso fue propiciado, junto con las necesidades demográficas de los estados, por la ascensión del poder y la influencia de la profesión médica, siendo los médicos higienistas los mayores divulgadores de normas y consejos, no solo para evitar los males físicos de las condiciones del trabajo sino 
a modificar hábitos y prácticas tradicionales de la vida privada donde los conocimientos y la experiencia de las mujeres siempre habían tenido lugar (Fuentes Caballero, 1996). La descarga de la práctica de la lactancia no supuso un problema, mientras se mantuvo en el ámbito privado, siendo favorecida por la influencia de comadronas, madres y amigas (Bolufer Peruga, 1992) (Fuentes Caballero, 1996). A partir del momento en que se contextualiza como un interés público, relacionado con el porvenir de la sociedad y la defensa de un modelo de familia, junto con la extensión de la lactancia asalariada a las clases trabajadoras (Bolufer Peruga, 1992), y la consideración de la nodriza como un oficio lucrativo cuando se contextualiza el problema (Fuentes Caballero, 1996); problema que se fundamenta en la estructura patriarcal jerarquizada de la época, siendo el colectivo masculino el que toma las decisiones (Soler, 2010) renegando del origen natural de la maternidad y desjerarquizando el hecho maternal del trabajo social, empobreciendo el rol de mujer madre, que se va a transmitir a los hijos (Sau, 1991). Es por ello que se va a establecer una categorización estereotipada de la figura de la nodriza, con un gran número de connotaciones negativas a nivel social $y$ moral, que incluso va a aparecer en la legislación reformista (Real Cédula sobre Expósitos de 20 de Enero de 1794) construyéndose un arquetipo de nodriza como mujer de baja extracción social, generalmente campesina, cuyas motivaciones económicas en el proceso natural supone falta de escrúpulos y que es capaz de elucubrar (al ocultar a los padres las enfermedades o accidentes del niños o su propia falta de leche) con el fin de no perder su fuente de ingresos fundamentándose la crítica al considerar que los cuidados maternos deben ser absolutamente desinteresados, pro- ductos del amor y del deber, y es el motivo por el que la sociedad patriarcal dominante justifica que una mujer asalariada no puede asumir las funciones maternas, reforzando esta idea al dar a entender que abandonan a sus propios hijos para atender a otros con un afán de lucro (Bolufer Peruga, 1992).

Dada la necesidad de mantener la supervivencia de los expósitos, en el siglo XIX, se iba a procurar la necesidad de apertura de Casas de Maternidad para la lactancia y reglamentar las condiciones de las nodrizas, que como indica Siles en su estudio sobre la industria de las nodrizas en Alicante, va a suponer la creación de instituciones dependientes de las diputaciones y ayuntamientos y al mismo tiempo una regulación de las condiciones de las lactantes (Siles González, La industria de las nodrizas en Alicante, 1868-1936, 1996). Previamente en nuestro país, la realeza durante el medievo seguía las indicaciones de Berdanio de Gordonio sobre las cualidades que debía tener una nodriza, y con el fin de mejorar la supervivencia de los herederos, las nodrizas dejaban a sus hijos al cargo de otras nodrizas, que eran sufragadas por el rey (Cabrera Sánchez, 2006). Al mismo tiempo indica la diferenciación en los niveles de supervivencia respecto a los niños de Instituciones por el insuficiente ratio de nodrizas (Llompart, 1979) (Quintana Andrés, 2003) y por las diferencias existentes entre las condiciones de las nodrizas en un mercado regulado por la oferta y la demanda en el que las nodrizas sanas y acomodadas eran contratadas por particulares que proporcionaban un salario más elevado, mientras que las más pobres eran las que trabajaban para las inclusas, hecho que se iba a relacionar con la peor consideración social de estas (Rodríguez Martín, 2008) (Campbell, 1989). Este proceso de instauración de una visión negativa de las nodri- 
zas por parte de la sociedad, propugnada por el intento de construcción de la visión de una "nueva madre" (Koutsoukos, 2009) junto con el desarrollo de las leches de fórmula provocan una sustitución gradual de las nodrizas por leches de fórmula (Stevens 1999), que supondrá cambios en el patrón de morbimortalidad infantil, que como indica Sartori alcanzará al $80 \%$ en niños alimentados de forma artificial en orfanatos durante los primeros años del siglo XX (Sartori, 2002). Al mismo tiempo, se la sociedad industrializada cambiará el modelo de alimentación infantil sin realizar una previsión previa de las consecuencias (aumento de hambruna, aumento de infecciones, etc.) y suponiendo un cambio social por la pérdida de la cultura de amamantamiento intergeneracional, que es un factor importante en la transmisión de los conocimientos madre-hija (Paricio, 2004). Este cambio se produce por diversos factores: mejoras en el desarrollo científico y en las condiciones de las leches artificiales, los intereses económicos de la industria y la creencia de los profesionales sanitarios de la lactancia artificial, pero sobre todo el proceso de liberación de la mujer de finales del siglo XIX, mujer que se incorpora al mercado laboral y ve la lactancia artificial como una mejora en la perspectiva de género que le permite abandonar la lactancia materna como símbolo de modernidad. No obstante a lo largo del siglo XX van a surgir voces que van a intentar defender los beneficios de la lactancia materna: Cicely Williams en el año 1933 atribuirá a la lactancia artificial miles de muertes en lactantes (Williams, Milk and Murder: Addres to the Rotary Club of Singapore in 1939, 1986) (Williams, A nutritional disease of childhood associated with a maize diet, 1933), la creación en 1956 de la Liga de leche, como grupo de apoyo a la lactancia, etc. Estos esfuerzos son respaldados por organizaciones internacionales, así en los objetivos del milenio de la OMS para el año 2000 (Organización Mundial de la Salud, 2003), la lactancia materna ha de ser un elemento clave para los sistemas sanitarios, recomendándose que en aquellas situaciones en las que los niños no puedan recibir leche de sus madres, la mejor opción es la leche donada (Hoddinott, Tappin, \& Wright, 2008). Tras la disminución de las nodrizas y ante la dificultad de asegurar leche materna por medio de nodrizas a niños enfermos o prematuros que por sus características requerían alimentación que no podía ser satisfecha mediante la lactancia artificial se crea en Viena en 1909 el primer banco de leche (Jones, 2003), (Neale, 1949), considerándose como una versión medicalizada de las nodrizas y demostrando su eficacia en la supervivencia de niños prematuros y en estado crítico, ventajas respecto al desarrollo psicomotor y la prevención antiinfecciosa entre otras (Verd Vallespir, Calvo Benito, Sáez Torres, \& Gayà Puig, 2003) (García Lara, NR, García-Algar, \& Pallás-Alonso, 2012), estando reconocidos los beneficios de la leche donada y las características a cumplir en el proceso de mantenimiento y distribución (Arnold L. , The cost-effectiveness of using banked donor milk in the neonatal intensive care unit: prevention of necrotizing enterocolitis, 2002). La implantación de los Bancos de Leche ha tenido un desarrollo desigual en los diferentes países en función de las características de las políticas de Salud Pública, (Springer, 2006) (Gutierrez \& Guera de Almeida, 1998) (Arnold L. , The cost-effectiveness of using banked donor milk in the neonatal intensive care unit: prevention of necrotizing enterocolitis, 2002), y el sistema de donaciones se inspira en una estética motivacional de tipo institucional que surge como consecuencia de las nuevas necesi- 
dades generadas por la incorporación de la mujer al sistema laboral y educativo y tras un proceso de concienciación comunitario que trasciende el apoyo de las redes espontáneas y vecinales de solidaridad, siendo el eslabón fundamental las donaciones maternas, facilitadas por el exceso de leche y el altruismo y que permiten a las donantes ir un paso más allá en el incremento de su autoestima y conciencia como mujer y madre producido por la lactancia (Leaf \& Winterson, 2009) (MartínezSabater, Siles-González, Escrivà-Aparisi, \& Ballestar-Tarín, 2014). La situación de la lactancia materna actualmente resulta de la evolución que ha seguido a lo largo del siglo XX en los países industrializados (MArtínez Fernández, y otros, 2004). Tras las revoluciones industriales y la incorporación de la mujer al sistema laboral y educativo se produce una transición de una estética natural tradicional, motivadora de la alimentación con leche natural (preferiblemente materna) cimentada en valores y sentimientos inspirados en la maternidad y la familia; a una estética industrial propulsora de la leche artificial en la que la motivación se sustenta en los valores y sentimientos enraizados en el profesionalismo y el tecnologicismo, que conlleva cambios incluso en los símbolos y el arte en el que se recogen las expresiones de sentimientos que se corresponden con la tendencia marcada por la estética natural: sentimiento de maternidad, lactancia como una encrucijada en la que se recogen los beneficios para la salud del niño, la salud de la madre y los efectos emocionales positivos para ambos. Posteriormente se produce un movimiento dialéctico que niega los valores tradicionales que limitan el papel de la mujer en la sociedad a poco menos que doméstica, cuidadora, procreadora y criadora "nutrix" de la prole, con la finalidad de poder integrarse en el plano laboral y dedicarle el tiempo y esfuerzo suficiente para alcanzar el profesionalismo (Siles, y otros, 1998). El feminismo, la democracia y el estado del bienestar de mediados de siglo XX impulsan valores de igualdad entre el hombre y la mujer en los que no cabe motivación alguna para la maternidad y la lactancia materna. Tras estos dos movimientos antitéticos se produce una síntesis de la mano del pensamiento postfeminista mediante el que algunas mujeres intentan rescatar algunos valores tradicionales abandonados ante las necesidades de profesionalización de la mujer y una sociedad más igualitaria a efectos de diferencias de género. Esta reinterpretación del papel de la mujer intenta conciliar la maternidad con el profesionalismo, pero esto implica una lucha para el reconocimiento de la profesional como madre y criadora y es, en este momento histórico, en el que se construye socialmente una nueva tipología de valores que es el resultado de la conjunción de los valores tradicionales y los nuevos: lactancia materna y lactancia natural entre los que caben el profesionalismo, el tecnologicismo, pero también el humanismo y la solidaridad comunitaria transmaternal (Siles González, La eterna guerra de la identidad enfermera: un enfoque dialéctico y deconstruccionista, 2005). Así, al igual que todos los procesos de cuidados han sufrido una transformación, las mujeres donantes a los bancos de leche suponen un "resurgir" de las nodrizas permitiendo la mejora de las tasas de supervivencia de los neonatos y los niños/as en estado crítico junto con las mejorar científicas en los procesos de la atención. No obstante, por otro lado, las nuevas estructuras familiares, la modificación de las conductas, etc. han hecho surgir en diferentes países y situaciones ofertas de venta de leche, junto con una proceso de normalización del 
intercambio con iniciativas al margen de los bancos de leche, utilizando los recursos que proporcionan las redes sociales e internet y que se convierte en una versión actual de la figura de las nodrizas (Akre, Gribble, \& Minchin, 2011).

Así, en resumen, respecto a la figura historia de la nodriza podemos indicar que supuso un elemento normalizado en la sociedad en el entorno de los cuidados con un rol importante en el patrón demográfico; pero dada la escasez progresiva de nodrizas en el ámbito institucional y la aceptación de mujeres con peores condiciones físicas y un nivel social más bajo lo que conllevó una caracterización negativa por parte de la sociedad que había sido vehiculizada por la prensa y la literatura divulgativa. Aunque durante el siglo XIX persistió una visión dual en la prensa en función del origen o el estatus del bebé alimentado: en las clases elevadas se valora la influencia en el seno familiar enfatizándose las cualidades aportadas en el seno familiar, y en los estamentos sociales más desfavorecidos, se incidió en la poca relación afectiva con los expósitos alimentados y la búsqueda del beneficio económico en la mercantilización de su leche. Esta visión negativa se acució por la crítica de la clases médica, que en el proceso de medicalización de los procesos biológicos, convierte a las nodrizas en objeto de sus críticas, las medicaliza y monopoliza el proceso de contratación y selección; y al mismo tiempo se produce una discriminación fruto de la concepción patriarcal de la sociedad y el carácter femenino de la actividad, acompañado de búsqueda de alternativas de alimentación infantil y la aparición de los productos farmacéuticos supone la aparición de campañas publicitarias que minusvaloran el rol y el oficio de nodriza, que hasta principios del siglo XX se había establecido como una fi- gura laboral normalizada, que profesionaliza su rol biológico a cambio de un salario, aunque en ocasiones, éste no iba a ser el único beneficio, ya que sobre todo en estatus elevadas se obtenían mejoras en las relaciones y en el rol social de la familia; por otro lado se establecían redes vecinales y de parentesco que se extendían durante años.

\section{TRABAJOS CITADOS}

- Akre, J; Gribble, K; \& Minchin, M. (2011) Milk sharing: from private practice to public pursuit. International Breatfeeding Journal 6(8).

- Arnold, D; \& Lockhart Borman, L. (1996) What are the Characteristics of the Ideal Human Milk Donor? J Hum Lact 12(143).

- Arnold, L. (2002) The cost-effectiveness of using banked donor milk in the neonatal intensive care unit: prevention of necrotizing enterocolitis. Journal of Human Lactation 18(2): 172-77.

- Arnold, L. (2006) Global health policies than support the use of banked donor human milk: a human rights issue. International Breastfeeding Journal 1(26).

- Ben-Lun, L. (Dec de 2006) Breastfeeding. The roots. Minerva Pediatr. 58(6): 551-556.

- Bolufer Peruga, M. (1992) Actitudes y discursos sobre la maternidad del siglo XVIII: la cuestión de la lactancia. Historia Social 14: 3-22.

- Botton Beja, F. (2000) China: su historia y cultura hasta 1800. El Colegio de México, México.

- Cabrera Sánchez, M. (2006) La transmisión del saber médico: la vida infantil en la edad media a través de los tratados pediátricos y de otras fuentes de la época. MERIDIES. Revista de Historia Medieval 8: 7-36.

- Campbell, L. (1989) Wet-nurses in early modern England: some evidence from the Townshend archive. Medical History 33: 360-370.

- Carrasquer, P; Toms, T; Tejero, E; \& Romero, A. (1998) El trabajo reproductivo. Papers 55: 95-114.

- Carvalho da Graça, L. C; Barbiéri Figuereido, M. D; \& Caetano Carreira, M. (2011) Contributions of the nur- 
sing intervention in primary healthcare for the promotion of breastfeeding. Rev. Latino-Am.Enfermagen 19(2).

- Dettwyler, K. (2004) When to Wean: Biological versus Cultural Perspectives. Clinica Obstetrics \& Gynecology 47(3): 712-713.

- Dirección General de Investigación y Salud Pública. (2005) Lactancia Materna. Recuperado el 12 de 03 de 2012, de Consellería de Sanitat: http://www.sp.san.gva. es/sscc/progSalud.jsp?CodProg=PS81\&Opcion=SAN MS11410

- Expósito González, R; Rubio Pilarte, J; \& Solórzano Sánchez, M. (14 de 06 de 2012) Recuperado el 01 de 03 de 2013, de Enfermería Avanza: http://enfeps.blogspot. com.es/2012/06/nodrizas.html

- Fildes, V. (1988) The English wet-nurse and her role in infant care 1538-1800. Medical History(32): 142-173.

- Fuentes Caballero, M. (1996) Ser nodriza en Barcelona: una posibilidad de supervivencia a final del siglo XIX. Políticas Feministas: ética, estética y feminismo: 81-93.

- García Lara, N.R; García-Algar, O; \& Pallás-Alonso, C. (2012) Sobre bancos de leche humana y lactancia materna. An Pediatr (Barc) 76(5): 247-249.

- Gutierrez, D; \& Guera de Almeida, J. (1998) Human milk banks in Brazil. J Hum Lact(14): 333-335.

Henderson, V. (1971) Principios básicos de los cuidados de enfermería. Consejo Internacional de Enfermería, Ginebra.

- Hernández Gamboa, E. (2008) Genealogía Histórica de la Lactancia Materna. Rev. Enfermería Actual en Costa Rica(15).

- Herrera Feria, M. (2003) La domesticación del cuerpo femenino en la perspectiva de la historia regional. Revista Géneros 10(29): 20-26.

- Hoddinott, P; Tappin, D; \& Wright, C. (2008) Clinical review: breastfeeding. $\mathrm{BMJ}(336)$ : 881-887.

- Instituto de Investigaciones Jurídicas de la UNAM. (1994) Diccionario Jurídico sobre Seguridad Social. UNAM, México.

- Jones, F. (2003) History of North American Donor Milk Banking: One hundred Years of Progress. Journal of $\mathrm{Hu}-$ man Lactation 19(3): 313-18.
- Koçtürk, T. (May de 2003) Foetal devopment and breastfeeding in early texts of the Islamic tradition. Acta Paeditr. 92(5): 617-620.

- Koutsoukos, S. (2009) "Mercenary wet-nurses": the discouse of medical doctors and portraits of the wet nurses Brazil in the second half of the 19th century. Hist Cienc Saude Manguinhos 16(2): 305-324.

- Larrañaga, I; Arregui, B; \& Arpal, J. (Mayo de 2004) El trabajo reproductivo o doméstico. Gaceta Sanitaria 18(1): 31-37.

- Leaf, A; \& Winterson, R. (2009) Reast-milk banking: evidence of benefit. J Paediatr Child Healt(19): 395-399.

- Llompart, G. (1979) La población hospitalaria y religiosa de Mallorca bajo el Rey Sancho (1311-13240. Cuadernos de Historia Jerónimo Zurita 33-34: 67-98.

- Macfarlane, A. (2002) Infant feeding in Japan. Recuperado el 12 de 03 de 2012, de http://www.alanmacfarlane. com/savage/INFANTS.PDF

- Mander, R. (2003) An analysis of Simpsons's notebook data on the wet nurse. Midwifery 19(19): 46-54.

- Martínez Fernández, M; Grifo Peñuelas, M; Canicatti Galiano, A; Sanz Rosado, A; Hindi, B; Kassis, E; \& Gonzalo Hombrados, J. (2004) Lactancia materna. Volviendo a los cincuenta. Semergen 30(4): 159-163.

- Martínez-Sabater, A; Siles-González, J; Escrivà-Aparisi, M; \& Ballestar-Tarín, M. (2014). Review of the Characteristica of Mothers Donor Milk Banks. American Journal of Nursing Research 2(1): 1-6.

- Moring, B. (1998) Motherhood, milk and money: infant mortality in pre-industrianl Findland. Soc Hist MEd 11(2): 177-196.

- Neale, A. (1949) Avances en Pediatría. Anales de medicina y cirugía 25(43): 38-50.

- Organización Mundial de la Salud. (2003) Estrategias para la alimentación del lactante y del niño pequeño. Recuperado el 21 de 03 de 2011, de Estrategías para la alimentación del lactante y del niño pequeño.: http:// www.who.int/nutrition/publications/gs_infant_feeding_text_spa.pdf

- Paricio Talayero, J. (2004) Tesis doctoral inédita leída en la Universidad Autónoma de Madrid. Facultad de Medicina. Departamento Epidemiología y Salud Pública. Fe- 
cha de lectura: 16 de Septiembre de 2004. Lactancia materna y hospitalización por infecciones en el primer año de vida. Universidad Autónoma de Madrid, Madrid.

- Paricio, J. (2004) Aspectos históricos de la alimentación al seno materno. En AEP, Guía para profesionales - Monografía de la Asociación Española de Pediatría 5. Ergón, Madrid: 7-21.

- Prado, R. (1902) Wet nurses. Their Hygienic Importance. Public Health Pap. Rep.(28): 307-310.

- Quintana Andrés, P. C. (2003) Grupos urbanos y asistencia social: el Hospital de San Martín de Las Palmas en el Seiscientos. VEGUETA(7): 41-64.

- Riverón Corteguera, R. (1995) Valor inmunológico de la leche materna. Rev Cubana Pediatr. 67(2).

- Rodríguez Martín, A. (2008) El destino de los niños de la inclusa de Pontevedra, 1872-1903. Cuaderno de Estudios Gallegos 15(121): 353-388.

- Ruf-Nabhan, E. (2000) N Book Review: Infants, Parents and Wet Nurses: Medieval Islamics Views on Breastfeeding and ther social implications. Journal of Human Lactation(16): 100 .

- Sarasúa, C. (1994) El servicio doméstico en la formación del mercado de trabajo madrileño, 1758-1868 (Primera ed.). S. XXI, Madrid.

- Sartori, E. (2002) Not only bottles. Glass baby's bottles in between the second half of XIXth century and the first decades of the XXth. Med Secoli 14(2): 609-622.

- Sau, V. (1991) La ética de la maternidad. En L. Luna, \& S. I. mujer (Ed.) Mujer y sociedad. Universitat de Barcelona, Barcelona: 1277-182.

- Siles González, J. (1996) La industria de las nodrizas en Alicante, 1868-1936. En S.Castillo El trabajo a través de la historia). UGT - Centro de Estudios Históricos. Asociación de Historia Social, Madrid:367-372.

- Siles González, J. (1996) Pasado, presente y futuro de la enfermería en España: perspectiva histórica y epistemológica. CECOVA, Alicante.

- Siles González, J. (2005) La eterna guerra de la identidad enfermera: un enfoque dialéctico y deconstruccionista. Index Enferm [online] 14(50).

- Siles González, J; \& Solano Ruiz, M. (2007) Estructuras sociales, división sexual del trabajo y enfoques metodo- lógicos: la estructura familiar y la función socio-sanitaria de la mujer. Investigación y educación en enfermería 25(1): 66-73.

- Siles, J; Gabaldón, E; Molero, D; Gallardo, Y; García Hernández, E; \& Galao, R. (1998) El eslabón biológico en la Historia de los Cuidados de Salud. El caso de las nodrizas (una visión antropológica de la enfermería). Index de Enfermería (edición digital) 20-21.

- Soler, E. (2010) Parentesco de leche y movilidad social. En P. Gonzalbo Aizpuru, Familias y relaciones diferenciales: género y edad (págs. 171-179) Universidad de Murcia. Servicio de Publicaciones, Murcia.

- Springer, S. (2006) Trends in human milk banking in Germany and Europe. Breastfeed Med, 26(1): 200-201.

- Stevens, E; Patrick, T; \& Pickler, R. (2009) A history of Infant feeding. J Perinat Educ 18(2) 32-39.

- Swain Müller, F; \& Aparecida Silva, I. (2009) Social representations abour support for breastfeeding in a group of breastfeeding women. Rev. Latino-Am.Enfermagen 17(5).

- Utrera Torres, M; Medina López, C; Vázquez Román, S; Alonso Díaz, C; Cruz-Rojo, J; Fernández Cooke, E; \& Pallás Alonso, C. (Mar de 2010) Does opening a milk bank in a neonatal unit change infant feeding practices? A before and after study. International Breastfeeding Journal 5(4).

- Verd Vallespir, S; Calvo Benito, J; Sáez Torres, C; \& Gayà Puig, T. (2003) Avances en el uso de la leche humana de donante. An Pediatr(Barc) 58(3).

- Williams, C. (December de 1933) A nutritional disease of childhood associated with a maize diet. Arch Dis Child, 48(8), 423-431.

- Williams, C. (1986) Milk and Murder: Addres to the Rotary Club of Singapore in 1939. IOCUROAP, Penang.

- Yalom, M. (1997) Historia del pecho (Primera ed.)Tusquets, Barcelona. 